January 30,2020

DAMTP-2011-101

\title{
Symmetry Breaking Bulk Effects in Local D-brane Models
}

\author{
Anshuman Maharana \\ DAMTP, University of Cambridge, \\ Wilberforce Road, Cambridge, \\ CB3 OWA, UK.
}

\begin{abstract}
We study symmetrybreaking effects in local D-brane models that arise as a result of compactification, focusing on models constructed on $\mathbb{C}^{3} / \mathbb{Z}_{3}$. Zero-modes of the Lichnerowicz operator in cone-like geometries have a power law behaviour; thus the leading symmetry breaking effects are captured by the modes with the lowest scaling dimension which transform non-trivially under the isometry group. Combining this with the fact that global symmetries in local models are gauged upon compactification we determine the strength and form of the leading operators responsible for the symmetry breaking.
\end{abstract}

electronic address: am794@cam.ac.uk 


\section{Introduction}

The bottom-up approach 1,10 is a promising direction for string phenomenology. In this approach the Standard Model degrees of freedom arise from a D-brane construction which is localised in the extra dimensions. Various properties of the Standard Model sector such as matter content, gauge couplings and Yukawa couplings can be computed in the local setting, without knowledge of the global aspects of the compactification. A modular approach is possible. After having constructed a realistic Standard Model sector in a local geometry one can then attempt to embed the model in a compactifiction. At the later stage one has to consider global aspects such as tadpole cancellation, moduli stabilisation, supersymmetry breaking and cosmology.

Isometries in the geometry imply global symmetries in the local model. Such symmetries can have phenomenologically interesting implications. For instance, it was shown in [11] that for the models constructed in [4,5] isometries of the local geometry imply that fermions in the lightest generation are massless at tree-level.

The fate of such symmetries once the local model is embedded in a compactification was discussed in [12]. The effect of compactification is to couple the model to gravity; theories of quantum gravity cannot possess exact continuous global symmetries 13 15. It was argued in 12 that once compactification effects are taken into account global symmetries in local models are gauged and the associated gauge field is a closed string (bulk) mode. Furthermore, compact Calabi Yaus with $S U(3)$ holonomy do not have any isometries. This implies that the gauge symmetry is spontaneously broken by the bulk. These effects are communicated to the open string sector via their interactions with the bulk modes. Once the bulk modes are integrated out one is left with an open string sector which has an approximate global symmetry, with the closed string sector providing the "flavon" vevs which parametrise the symmetry breaking. The strength of symmetry breaking necessarily scales as an inverse power of the compactification volume; as in the infinite volume limit Newton's constant vanishes and

the global symmetry is restored. Couplings in the Standard Model sector which vanish in the non-compact analysis due to the presence of the global symmetry can take on values which scale as inverse powers of the volume; if the volume of the compactification is large one can 
generate parametrically small couplings in the Standard Model sector.

The $\mathbb{C}^{3} / \mathbb{Z}_{3}$ geometry provides an attractive starting point 1 for local model building. Models on complex cones over the lower del Pezzo surfaces [4 6] are closely related, in order to obtain the Standard Model gauge groups at low energies one has to Higgs these models to $\mathbb{C}^{3} / \mathbb{Z}_{3}$. The geometry has a $S U(3) \times U(1)$ isometry. In this paper we shall focus on $\mathbb{C}^{3} / \mathbb{Z}_{3}$ (either singular or resolved); and compute the volume dependence and form of the leading operators that break the $S U(3) \times U(1)$ symmetry as a result of compactification. We shall do so by determining the leading deviations in the metric which break the isometries once the geometry is glued on to a bulk to form a compact space. These can be obtained by carrying out a small fluctuation analysis in the local geometry, along the lines of [16 18]. For simplicity, we will model the effect of compactification on the local geometry via a deformation which preserves the Ricci flatness condition. However, general supersymmetry-breaking deformations will not preserve Ricci flatness, and a systematic study of supersymmetry-breaking effects requires the analysis of other modes such as the fluxes and their backreaction on the metric. We hope to return to these issues in the future.

In the region where the deformation is small compared to the background metric, a Ricci flat deformation can be written as a series in zero-modes of the Lichnerowicz operator constructed from the local geometry. For cone-like geometries zero-modes of the Lichnerowicz operator have angular dependence given by symmetric tensor harmonics on the base space and a power law radial behaviour. Thus,

$$
\delta g_{i j}=\sum_{I} c_{I} Y_{i j}^{I}(\psi) r^{\Delta_{I}}
$$

where $Y_{i j}^{I}$ are harmonics on the base, $\Delta_{I}$ the radial scaling associated with the harmonic $Y_{i j}^{I}$ and $c_{I}$ Fourier coefficients. Modes with positive $\Delta_{I}$ grow in the asymptotic end of the cone and fall off in the tip region: these are induced as a result of compactification 1 , In the bulk of the compactification the isometries are badly broken, the deformed non-compact metric smoothly connects onto this "generic" metric; we therefore will assume that in the asymptotic region of the cone all terms in the sum (1.1) are of equal magnitude. Given this, the power law behaviour in the radial direction implies that the perturbation in the vicinity of the tip of the

\footnotetext{
${ }^{1}$ Modes with negative $\Delta_{I}$ have profiles that vanish in the asymptotic region and are to be associated with blow-up modes.
} 
cone is well approximated by the modes with the smallest positive values of $\Delta_{I}$. Thus in order to obtain the leading effects that break a certain isometry one has to isolate the harmonic $Y_{i j}^{I}$ in the expansion (1.1) with the smallest positive value of $\Delta_{I}$ which is not a singlet under the action of the isometry group.

Once we have isolated the relevant harmonics we shall construct the lowest dimension operators that involve both the Standard Model sector and the symmetry breaking harmonics (flavons). These operators have to be gauge invariant under the action of the isometry group; this will allow us to enumerate the form of the symmetry breaking operators that arise in the open string sector.

\section{Perturbations and Breaking of Isometries on $\mathbb{C}^{3} / \mathbb{Z}_{3}$}

The space $\mathbb{C}^{3} / \mathbb{Z}_{3}$ is obtained from $\mathbb{C}^{3}$ by making the identification $z^{i} \equiv e^{2 \pi i / 3} z^{i}$. The space inherits the Kähler potential of $\mathbb{C}^{3}, K=z^{i} \bar{z}_{\bar{i}}=r^{2}$. The identification breaks the $S O(6)$ isometry of $\mathbb{C}^{3}$ to $U(3) \cong S U(3) \times U(1)$. Under the action of the isometry group the complex coordinates $z^{i}$ transform in the fundamental, $z^{i} \rightarrow U^{i}{ }_{j}{ }^{j}$.

The origin is a fixed point of the orbifold action and is singular. The singularity can be resolved [19] by modifying the Kähler potential to

$$
r^{2} K^{\prime}\left(r^{2}\right)=\left(r^{6}+r_{0}^{6}\right)^{1 / 3}
$$

The metric on the space then becomes

$$
g_{m \bar{n}}=\frac{\left(r^{6}+r_{0}^{6}\right)^{1 / 3}}{r^{2}} \delta_{m \bar{n}}-\frac{r_{0}^{6}}{r^{4}\left(r^{6}+r_{0}^{6}\right)^{2 / 3}} z_{m} z_{\bar{n}},
$$

the locus $r=0$ now corresponds a finite size $\mathbb{P}^{2}$ with the canonical Fubini Study metric of radius $r_{0}$.

\section{Perturbation Analysis}

In order to discuss perturbations it is useful to consider $\mathbb{C}^{3} / \mathbb{Z}_{3}$ as a cone with base $S^{5} / \mathbb{Z}_{3}$. Static perturbations of general CY cones

$$
d s_{6}^{2}=g_{m n} d y^{m} d y^{n}=d r^{2}+r^{2} d s_{\mathcal{B}_{5}}^{2}=d r^{2}+r^{2} \tilde{g}_{i j} d \Psi^{i} d \Psi^{j}
$$


were discussed in 18, the results of which we now review. For metric perturbations, $\delta g_{m n}=$ $h_{m n}$, linearized Einstein equations are given by $\Delta_{K} h_{m n}=0$, where $\Delta_{K}$ is the Lichnerowicz operator constructed from the metric $g_{m n}$. Working in transverse gauge with respect to the base, $\tilde{\nabla}^{i} h_{i j}=\tilde{\nabla}^{i} h_{i r}=0$; Einstein equations imply $h_{r i}=h_{r r}=0$. The angular components of the metric are required to take the form

$$
h_{i j}=\sum_{I_{t}} \phi^{I_{t}}(r) Y_{i j}^{I_{t}}(\Psi)
$$

with the index $I^{t}$ running over the symmetric traceless two tensor harmonics on the base $\mathcal{B}_{5}$. These are eigenfunctions of the Lichnerowicz operator constructed from the base metric $\tilde{g}_{i j}$. The functions $\phi^{I_{t}}(r)$ are solutions of the differential equation

$$
\left(\partial_{r}^{2}-\frac{4}{r} \partial_{r}+\frac{4-\lambda^{I_{t}}}{r^{2}}\right) \phi^{I_{t}}=0
$$

where $\lambda^{I_{t}}$ is the eigenvalue of the harmonic $Y_{i j}^{I_{t}}$. The general solution of which is

$$
\phi^{I_{t}}(r)=a_{+}^{I_{t}} r^{+\Delta}+a_{-}^{I_{t}} r^{-\Delta}
$$

with

$$
\Delta=\sqrt{\lambda^{I_{t}}-4}
$$

where $a_{ \pm}^{I_{t}}$ are integration constants, fixed by the boundary conditions.

\section{Tensor Harmonics on $S^{5} / \mathbb{Z}_{3}$}

The tensor harmonics on $S^{5} / \mathbb{Z}_{3}$ can be obtained from those on $S^{5}$ by carrying out an orbifold projection. We begin by discussing some basic properties of tensor harmonics on d-spheres (see for e.g. [20]). Tensor harmonics on the d-sphere can be constructed by considering functions of the form

$$
\begin{aligned}
& Y_{i j}^{l}=C_{A_{1} \ldots . . A_{l} ; B_{1} B_{2}}^{l} \Omega^{A_{1}} \ldots . \Omega^{A_{2}} \hat{D}_{i} \Omega^{B_{1}} \hat{D}_{j} \Omega^{B_{2}}, \quad l \geq 2 \\
& A_{1}, \ldots ., A_{l}, B_{1}, B_{2}=1 \ldots d+1
\end{aligned}
$$

where $\Omega^{A}$ are homogeneous coordinates on the d-sphere $\Omega^{A} \cdot \Omega^{A}=1$ and $\hat{D}$ denotes the covariant derivative compatible with the $\mathrm{d}$-sphere. $C_{A_{1} \ldots . . A_{l} ; B_{1} B_{2}}^{l}$ is a constant tensor which is 
Table 1: Tensor Harmonics on $S^{5} / \mathbb{Z}_{3}$ for $l \leq 4$.

\begin{tabular}{|c|c|}
\hline$l$ & $\begin{array}{l}S U(3) \text { Representations } \\
\text { and } U(1) \text { charge }\end{array}$ \\
\hline 2 & $(0,0)^{0},(1,1)^{0},(2,2)^{0}$ \\
\hline 3 & $\begin{array}{l}(1,1)^{-3},(3,0)^{-3},(2,2)^{-3} \\
(1,1)^{+3},(0,3)^{+3},(2,2)^{+3}\end{array}$ \\
\hline 4 & $\begin{array}{l}(1,1)^{0},(3,0)^{0},(0,3)^{0} \\
(2,2)^{0},(1,4)^{0},(4,1)^{0} \\
(3,3)^{0},(1,2)^{-6},(2,1)^{+6}\end{array}$ \\
\hline
\end{tabular}

symmetric and traceless with respect to the indices $A_{i}$ and $B_{j}$ and satisfies $C_{\left(A_{1} \ldots . . A_{l} ; A_{l+1}\right)}^{B}=$ $C_{\left(A_{1} \ldots . . A_{l} ; A_{l+1}\right)}^{B}=0$. For a fixed value of $l$ the harmonics transform as an irreducible representation of $S O(d+1)$. For the five sphere the associated highest weight state has Dynkin label $(l-2,2,2)$. The eigenvalue for the Lichnerowicz operator is

$$
\lambda^{I_{t}}=l(l+4)+8
$$

Equation 2.7 then gives

$$
\Delta=l+2 .
$$

In order to obtain the harmonics on $S^{5} / \mathbb{Z}_{3}$ we decompose the $S O(6)$ representations associated with the harmonics of $S^{5}$ under the maximal subgroup $S U(3) \times U(1)$ and project to states that have vanishing $\mathrm{U}(1)$ charge ${ }^{2}$ modulo 3 . Table 1 lists the $S U(3)$ representation and $\mathrm{U}(1)$ charge of the harmonics for $l \leq 4$. Details of the decomposition are given in the appendix.

\section{Strength of Isometry Breaking Effects}

As discussed in the introduction, in the vicinity of the tip of the cone the leading isometry breaking effects are captured by the harmonics which have the lowest (positive) scaling dimension and transform non-trivially under the action of the isometry group 3 In associating

\footnotetext{
${ }^{2}$ We normalise the $U(1)$ charge so that the vector of $S O(6)$ decomposes into the fundamental and antifundamental of $S U(3)$ with charge +1 and -1 .

${ }^{3}$ The singlet at $l=2$ with $\Delta=-4$ is related to the resolution 2.1. The associated mode with a vanishing profile in the asymptotic end has a fall off $r^{-4}$, i.e. is suppressed by $r^{-6}$ relative to the background metric. This is in keeping with the small $r_{0}$ expansion of 2.1 .
} 
the effects of compactification only with modes with positive scaling, we are assuming that there are no compactification effects that grow in the small $\mathrm{r}$ region. In general such effects can be present - the analogue of relevant deformations in AdS/CFT examples. The presence of such modes indicates that the small $\mathrm{r}$ geometry is unstable to certain deformations of the bulk. One would then like to find symmetry reasons to prevent these unstable modes to be turned; so that the geometry in small $\mathrm{r}$ (location of the brane set up) is stable. For the $S U(3)$ generators, Table 1 shows that these harmonics are at $l=2(\Delta=4)$ with $S U(3)$ Dynkin label $(1,1)$ and $(2,2)$. The background metric 2.3) has angular components which scale as $r^{2}$; thus in the vicinity of the tip of the cone $\Delta=4$ deformations are suppressed by a factor $\left(r / r_{\text {asym }}\right)^{2}$ relative to the background (where $r_{\text {asym }}$ is the value of the radial coordinate at which the cone is glued on to the bulk). For an open string sector which is localised on a $\mathbb{P}^{2}$ resolving the singularity at $r=0$, the relevant scale at small $r$ is the resolution radius $r_{0}$. In the absence of any anisotropies in the compactification $r_{\text {asym }}$ is of the same scale as the compactification radius. Thus compactification effects that break the $S U(3)$ symmetry are of magnitude

$$
\epsilon \sim\left(\frac{r_{0}}{r_{\text {asym }}}\right)^{2} \sim\left(\frac{\tau_{s}}{\tau_{b}}\right)^{1 / 2} .
$$

Where $\tau_{s}$ and $\tau_{b}$ are the Kähler moduli associated with the blow-up mode and the volume modulus. For models on the singular locus the relevant scale at small $r$ is the string scale, thus

$$
\epsilon \sim \frac{1}{\mathcal{V}^{1 / 3}}
$$

Where $\mathcal{V}$ is the volume of the compactification in string units.

The $U(1)$ symmetry is unbroken at $l=2$; it is broken by $l=3$ modes with charge \pm 3 . The associated suppression factor is

$$
\delta \sim \frac{1}{\mathcal{V}^{1 / 2}} .
$$

Thus at large volume the $S U(3)$ and $U(1)$ symmetry breaking parameters are hierarchically separated.

\section{Symmetry Breaking Operators for D3-branes at $\mathbb{C}^{3} / \mathbb{Z}_{3}$}

In this section we shall consider D3-branes at a $\mathbb{C}^{3} / \mathbb{Z}_{3}$ singularity and determine the form of the symmetry breaking operators in the open string sector that are induced as a result of 


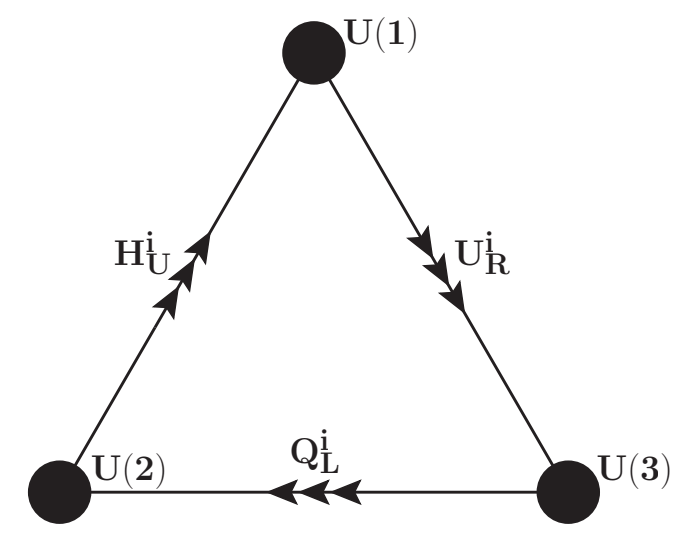

Figure 1: Quiver diagram for D3-branes probing a $\mathbb{C}^{3} / \mathbb{Z}_{3}$ singularity.

compactification. Our guiding principle will be gauge invariance under the flavon (isometry) group.

The massless spectrum of D3-branes probing a $\mathbb{C}^{3} / \mathbb{Z}_{3}$ singularity is summarised in Figure 1 by a quiver diagram; gauge groups are indicated by nodes and bifundamental chiral multiplets are indicated by arrows between the nodes. Each chiral multiplet has a threefold degeneracy. This provides the generation index for the models in [1,4,5. As shown in the figure, the chiral multiplets are identified with the left-handed quarks $\left(Q_{L}^{i}\right)$, the right-handed up quarks $\left(U_{R}^{i}\right)$ and the up Higgs $\left(H_{u}^{i}\right)$. They interact via a Yukawa interaction

$$
\epsilon_{i j k} H_{u}^{i} Q_{L}^{j} U_{R}^{k}
$$

invariant under the isometry group $S U(3) \times U(1)$ with $H_{U}^{i}, Q_{L}^{i}$ and $U_{R}^{i}$ transforming in the fundamental.

In order to obtain the form of the leading operators which break the symmetry in the Yukawa interactions, recall that the product of three fundamentals $\left(H_{U}^{i}, Q_{L}^{i}, U_{R}^{i}\right)$ decomposes into irreducibles as

$$
(1,0) \times(1,0) \times(1,0) \rightarrow(0,0) \oplus(1,1) \oplus(1,1) \oplus(3,0)
$$

In section 2 we found that the metric fluctuations with the smallest scaling dimension which break the $S U(3)$ symmetry (flavons vevs) transform as $(1,1)$ and $(2,2)$. The operators which 
break the symmetry in the open string sector are gauge singlets that can be formed from the product of the flavon vevs with the irreducible representations that appear in the decomposition (3.2). Such singlets arise from the product ${ }^{4}$

$$
(1,1) \times(1,1) \rightarrow(0,0) \oplus(1,1) \oplus(1,1) \oplus(2,2) \oplus(3,0)
$$

Thus once the bulk modes are integrated out the leading symmetry breaking operators that can arise in the open string sector are given by the $(1,1) \mathrm{s}$ in $(3.2)$. These are

$$
\begin{aligned}
\lambda_{j}{ }^{i} & =\epsilon_{j m n} H_{U}^{(i} Q_{L}^{m)} U_{R}^{n}, \\
\beta_{j}{ }^{i} & =\epsilon_{j m n} H_{U}^{m} Q_{L}^{n} U_{R}^{i}-\frac{1}{3} \delta_{j}{ }^{i} \epsilon_{m n p} H_{U}^{m} Q_{L}^{n} U_{R}^{p} .
\end{aligned}
$$

The volume dependence of the strength of these operators is given by that of the flavon vev 2.12 , i.e. $\mathcal{V}^{-1 / 3}$. It will be interesting to perform the complimentary analysis of a CFT computation involving three open string states and a bulk KK mode in the $T^{6} / \mathbb{Z}_{3}$ toriodal orbifold and infer the pattern of symmetry breaking in the open string sector from the amplitude.

\section{Discussion}

Central to our analysis has been the assumption that at the asymptotic end of the cone all terms in the expansion (1.1) are of equal magnitude. This assumption can be checked by exploiting the progress in computing numerical metrics on Calabi-Yau manifolds 21, 22. A one parameter family of Ricci flat metrics on $K 3$ was obtained in 21 by starting from the orbifold $\mathrm{T}^{4} / \mathbb{Z}_{2}$, and resolving the singular points to a finite size two sphere. In the large volume limit the local geometry near the resolutions is given by the Eguchi-Hanson geometry with corrections associated with finite volume effects. The numerical metric provides a setting in which one has explicit knowledge of the Fourier coefficients $c_{I}$, can be used to check the reliability of the assumptions on the size of the Fourier coefficients.

We have confined our attention to breaking of isometries as result of metric perturbations. A compactification with moduli stabilisation is going to have other fields such as fluxes and a non-trivial warp factor which can also lead to symmetry breaking. In the analysis of realistic

\footnotetext{
${ }^{4}$ The are no singlets in the tensor products $(1,1) \times(3,0),(2,2) \times(1,1)$ and $(2,2) \times(3,0)$.
} 
models one has to include these effects. Each mode would have an associated SU(3) representation. For instance the warp factor satisfies the scalar Laplacian equation in the extra dimensions and transforms with $\mathrm{SO}(6)$ Dynkin label $(l, 0,0)$. Furthermore, as was emphasised in the context of brane inflation in [17], the leading physical effect can arise in second-order perturbation theory. Reference [23] provides the back-reacted geometry of $\mathbb{C}^{3} / \mathbb{Z}_{3}$ with the blow-up mode stabilised by gaugino condensation on D7-branes. The geometry has a $S U(3)$ isometry.

\section{Conclusions}

In this paper we have studied the symmetry breaking effects that arise as a result of compactification in local models on $\mathbb{C}^{3} / \mathbb{Z}_{3}$. The "power-law filtering" of modes in cone-like geometries combined with the requirement of gauge invariance under the flavon group allowed us to obtain the strength and form of the leading operators responsible for the symmetry breaking. At large volume we found a hierarchical separation in the breaking parameters for the $S U(3)$ and $U(1)$ symmetries.

Although our focus has been on models on $\mathbb{C}^{3} / \mathbb{Z}_{3}$, the approach is quite general and should be useful in understanding how symmetry breaking effects in the bulk are communicated to the open string sector in local models. It will be interesting to carry out a similar analysis for models on complex cones over del Pezzo surfaces $5[3,6]$ and F-theory models [8,9] and study implications for flavour structure.

\section{Acknowledgments}

I would like to thank Maciej Dunajski, Thomas Grimm, Liam McAllister, Fernando Quevedo and Ashoke Sen for very helpful communication and discussion. AM is funded by the European Union under the Seventh Framework Programme (FP7) and the University of Cambridge.

\footnotetext{
${ }^{5}$ These geometries have a $E_{n}$ global symmetry 24 .
} 


\section{Appendix}

\section{Decomposition of tensor harmonics on $\mathrm{S}^{5}$ under $\mathrm{SU}(3) \times \mathrm{U}(\mathbf{1})$}

As discussed in section 2 tensor harmonics on the the five sphere are labelled by a single quantum number $l(\geq 2)$, with the highest weight state having $S O(6)$ Dynkin label $(l-2,2,2)$. In this appendix we decompose the $l=2,3$ and 4 representations under $S U(3) \times U(1)$.

- $l=2$

$$
\begin{aligned}
(0,2,2) \rightarrow \quad & (2,0)^{-4} \oplus(1,0)^{-2} \oplus(2,1)^{-2} \oplus \\
& (0,0)^{0} \oplus(1,1)^{0} \oplus(2,2)^{0} \oplus \\
& (1,2)^{+2} \oplus(0,1)^{+2} \oplus(0,2)^{+4}
\end{aligned}
$$

- $l=3$

$$
\begin{aligned}
(1,2,2) \rightarrow \quad & (2,1)^{-5} \oplus(1,1)^{-3} \oplus(3,0)^{-3} \oplus(2,2)^{-3} \oplus \\
& (0,1)^{-1} \oplus(2,0)^{-1} \oplus(1,2)^{-1} \oplus(3,1)^{-1} \oplus(2,3)^{-1} \oplus \\
& (3,2)^{+1} \oplus(1,3)^{+1} \oplus(2,1)^{+1} \oplus(0,2)^{+1} \oplus(1,0)^{+1} \oplus \\
& (2,2)^{+3} \oplus(0,3)^{+3} \oplus(1,1)^{+3} \oplus(1,2)^{+5}
\end{aligned}
$$

- $l=4$

$$
\begin{aligned}
(2,2,2) \rightarrow \quad & (2,2)^{-6} \oplus(1,2)^{-4} \oplus(3,1)^{-4} \oplus(2,3)^{-4} \oplus \\
& (0,2)^{-2} \oplus(2,1)^{-2} \oplus(1,3)^{-2} \oplus(4,0)^{-2} \oplus(3,2)^{-2} \oplus(2,4)^{-2} \oplus \\
& (0,3)^{0} \oplus(4,1)^{0} \oplus(1,1)^{0} \oplus(2,2)^{0} \oplus(3,3)^{0} \oplus(1,4)^{0} \oplus(3,0)^{0} \oplus \\
& (4,2)^{+2} \oplus(2,3)^{+2} \oplus(0,4)^{+2} \oplus(3,1)^{+2} \oplus(1,2)^{+2} \oplus(2,0)^{+2} \oplus \\
& (3,2)^{4} \oplus(1,3)^{4} \oplus(2,1)^{4} \oplus(2,2)^{6}
\end{aligned}
$$

\section{References}

[1] G. Aldazabal, L. E. Ibanez, F. Quevedo and A. M. Uranga, "D-branes at singularities: A Bottom up approach to the string embedding of the standard model,” JHEP 0008 (2000) 002 arXiv:hep-th/0005067]. 
[2] G. Aldazabal, L. E. Ibanez, F. Quevedo and A. M. Uranga, "From branes at singularities to particle physics," Prepared for 9th Marcel Grossmann Meeting on Recent Developments in Theoretical and Experimental General Relativity, Gravitation and Relativistic Field Theories (MG 9), Rome, Italy, 2-9 Jul 2000

I. Antoniadis, E. Kiritsis and T. N. Tomaras, "A D-brane alternative to unification," Phys. Lett. B 486 (2000) 186 arXiv:hep-ph/0004214.

D. Berenstein, V. Jejjala and R. G. Leigh, "The Standard model on a D-brane," Phys. Rev. Lett. 88 (2002) 071602 arXiv:hep-ph/0105042.

J. F. G. Cascales, M. P. Garcia del Moral, F. Quevedo, A. M. Uranga, "Realistic D-brane models on warped throats: Fluxes, hierarchies and moduli stabilization," JHEP 0402 (2004) 031. hep-th/0312051.

[3] H. Verlinde and M. Wijnholt, "Building the standard model on a D3-brane," JHEP 0701 (2007) 106 arXiv:hep-th/0508089.

[4] J. P. Conlon, A. Maharana, F. Quevedo, "Towards Realistic String Vacua," JHEP 0905, 109 (2009). arXiv:0810.5660 [hep-th]].

[5] S. Krippendorf, M. J. Dolan, A. Maharana, F. Quevedo, "D-branes at Toric Singularities: Model Building, Yukawa Couplings and Flavour Physics," JHEP 1006 (2010) 092. arXiv:1002.1790 [hep-th]].

[6] M. J. Dolan, S. Krippendorf, F. Quevedo, "Towards a Systematic Construction of Realistic D-brane Models on a del Pezzo Singularity," [arXiv:1106.6039 [hep-th]].

[7] V. Balasubramanian, P. Berglund, I. Garcia-Etxebarria, "Toric Lego: A Method for modular model building," JHEP 1001 (2010) 076. [arXiv:0910.3616 [hep-th]].

[8] C. Beasley, J. J. Heckman, C. Vafa, "GUTs and Exceptional Branes in F-theory - I," JHEP 0901 (2009) 058. arXiv:0802.3391 [hep-th]].

R. Donagi, M. Wijnholt, "Model Building with F-Theory," arXiv:0802.2969 [hep-th]].

C. Beasley, J. J. Heckman, C. Vafa, "GUTs and Exceptional Branes in F-theory - II: Experimental Predictions," JHEP 0901 (2009) 059. [arXiv:0806.0102 [hep-th]]. 
[9] H. Hayashi, T. Kawano, R. Tatar and T. Watari, "Codimension-3 Singularities and Yukawa Couplings in F-theory," Nucl. Phys. B 823 (2009) 47 [arXiv:0901.4941 [hep-th]]. R. Blumenhagen, T. W. Grimm, B. Jurke, T. Weigand, "F-theory uplifts and GUTs," JHEP 0909 (2009) 053. arXiv:0906.0013 [hep-th]].

S. Cecotti, M. C. N. Cheng, J. J. Heckman, C. Vafa, "Yukawa Couplings in F-theory and Non-Commutative Geometry," arXiv:0910.0477 [hep-th]].

J. P. Conlon, E. Palti, "Aspects of Flavour and Supersymmetry in F-theory GUTs," JHEP 1001 (2010) 029. arXiv:0910.2413 [hep-th]].

E. Dudas, E. Palti, "Froggatt-Nielsen models from E(8) in F-theory GUTs," JHEP 1001 (2010) 127. arXiv:0912.0853 [hep-th]].

L. Aparicio, A. Font, L. E. Ibanez, F. Marchesano, "Flux and Instanton Effects in Local F-theory Models and Hierarchical Fermion Masses," arXiv:1104.2609 [hep-th]].

M. J. Dolan, J. Marsano, N. Saulina, S. Schafer-Nameki, "F-theory GUTs with U(1) Symmetries: Generalities and Survey," arXiv:1102.0290 [hep-th]].

[10] F. Marchesano, P. McGuirk and G. Shiu, "Chiral matter wavefunctions in warped compactifications," JHEP 1105 (2011) 090 arXiv:1012.2759 [hep-th]].

[11] C. P. Burgess, S. Krippendorf, A. Maharana, F. Quevedo, "Radiative Fermion Masses in Local D-Brane Models," JHEP 1105 (2011) 103. arXiv:1102.1973 [hep-th]].

[12] C. P. Burgess, J. P. Conlon, L. Y. Hung, C. H. Kom, A. Maharana and F. Quevedo, "Continuous Global Symmetries and Hyperweak Interactions in String Compactifications," JHEP 0807 (2008) 073 arXiv:0805.4037 [hep-th]].

[13] T. Banks, L. J. Dixon, "Constraints on String Vacua with Space-Time Supersymmetry," Nucl. Phys. B307 (1988) 93-108.

[14] T. Banks, N. Seiberg, "Symmetries and Strings in Field Theory and Gravity," Phys. Rev. D83 (2011) 084019. arXiv:1011.5120 [hep-th]]. 
[15] S. Hellerman, E. Sharpe, "Sums over topological sectors and quantization of FayetIliopoulos parameters," arXiv:1012.5999 [hep-th]].

[16] O. Aharony, Y. E. Antebi and M. Berkooz, "Open string moduli in KKLT compactifications," Phys. Rev. D 72, 106009 (2005) arXiv:hep-th/0508080.

[17] D. Baumann, A. Dymarsky, S. Kachru, I. R. Klebanov and L. McAllister, "Holographic Systematics of D-brane Inflation," JHEP 0903, 093 (2009) arXiv:0808.2811 [hep-th]]. D. Baumann, A. Dymarsky, S. Kachru, I. R. Klebanov and L. McAllister, "D3-brane Potentials from Fluxes in AdS/CFT," JHEP 1006, 072 (2010) arXiv:1001.5028 [hep-th]].

[18] S. Gandhi, L. McAllister and S. Sjors, "A Toolkit for Perturbing Flux Compactifications," arXiv:1106.0002 [hep-th].

[19] C. A. Lutken, "Geometry Of The Z Fold," J. Phys. A A21 (1988) 1889.

[20] A. Ishibashi and H. Kodama, "Perturbations and Stability of Static Black Holes in Higher Dimensions," arXiv:1103.6148 [hep-th].

P. Kanti, H. Kodama, R. A. Konoplya, N. Pappas, A. Zhidenko, "Graviton Emission in the Bulk by a Simply Rotating Black Hole," Phys. Rev. D80 (2009) 084016. arXiv:0906.3845 [hep-th]].

[21] M. Headrick and T. Wiseman, "Numerical Ricci-flat metrics on K3," Class. Quant. Grav. 22 (2005) 4931 arXiv:hep-th/0506129.

[22] M. R. Douglas, R. L. Karp, S. Lukic and R. Reinbacher, "Numerical Calabi-Yau metrics," J. Math. Phys. 49 (2008) 032302 arXiv:hep-th/0612075].

[23] B. Heidenreich, L. McAllister, G. Torroba, "Dynamic SU(2) Structure from Seven-branes," JHEP 1105 (2011) 110. arXiv:1011.3510 [hep-th]].

[24] S. Franco, A. Hanany, P. Kazakopoulos, "Hidden exceptional global symmetries in 4-D CFTs," JHEP 0407 (2004) 060. hep-th/0404065. 